Table: Baseline characteristics of JSLE patients in the Arthritis UK Centre for Adolescent Rheumatology

\begin{tabular}{lccc}
\hline & CKD & Without CKD & $\mathrm{p}$ \\
\hline Number of patients, $\mathrm{n}(\%)$ & $17(39 \%)$ & $27(61 \%)$ & \\
Female, $\mathrm{n}(\%)$ & $14(80 \%)$ & $23(85.2 \%)$ & $\mathrm{p}=0.47$ \\
Age at diagnosis, years & $12(10-11)$ & $12(11-15)$ & $\mathrm{p}=0.62$ \\
Disease duration, years & $12(8-14)$ & $12(7-13)$ & $\mathrm{p}=0.78$ \\
Highest dsDNA & $215(34-644)$ & $32(6.8-104)$ & $\mathrm{p}=0.03^{*}$ \\
SLEDAI at last assessment & $2(0-4)$ & $0(0-2)$ & $\mathrm{p}=0.33$ \\
Arthritis, $\mathrm{n}(\%)$ & $13(76 \%)$ & $16(59 \%)$ & $\mathrm{p}=0.24$ \\
Rituximab, number of courses & $1.5(0-2.3)$ & $0(0-1)$ & $\mathrm{p}=0.04^{*}$ \\
Mycophenolate Mofetil, months & $44(21-96)$ & $17(0-70)$ & $\mathrm{p}=0.04^{*}$ \\
Steroids, months & $48(37-82)$ & $25(11-50)$ & $\mathrm{p}=0.03^{*}$ \\
Numbers are medians (interquartile ranges) unless otherwise stated. ${ }^{*} \mathrm{p}<0.05$ is significant \\
\hline
\end{tabular}

Disclosure of Interests: None declared

DOI: 10.1136/annrheumdis-2019-eular.5143

\section{SAT0481 DIAGNOSIS AND INITIAL MANAGEMENT OF JUVENILE- ONSET SYSTEMIC LUPUS ERYTHEMATOSUS IN THE UK AND IRELAND}

Hanna Lythgoe ${ }^{1}$, Clare Pain ${ }^{2}$, Eve Smith ${ }^{1,2}$, Michael Beresford ${ }^{1,2}{ }^{1}$ University of Liverpool, Department of Women's and Children's Health, Institute of Translational Medicine, Liverpool, United Kingdom; ${ }^{2}$ Alder Hey Children's NHS Foundation Trust, Department of Paediatric Rheumatology, Liverpool, United Kingdom

Background: The incidence of Juvenile-onset Systemic Lupus Erythematosus (JSLE) in the UK is not well described. Furthermore, we do not know how children and young people initially present and when and where they access care. Previous work has described significant variation in time to diagnosis for UK patients ${ }^{1}$.

Objectives: To describe how patients with JSLE currently present, are diagnosed and managed across the UK

Methods: Data was collected over 13 months on all children (aged $<18$ years) in the UK and Ireland with a new diagnosis of JSLE (meeting either American College of Rheumatology classification criteria (ACR-1997) or Systemic Lupus International Collaborating Clinics classification criteria (SLICC-2012)). Data was collected monthly from all UK and Ireland paediatricians using British Paediatric Surveillance Unit (BPSU) methodology ${ }^{2}$ and from relevant adult clinicians using a parallel reporting system. Anonymised patient data was collected by the clinician and sent to the study team. Patient consent was not required following Ethical and Confidentiality Advisory Group approval.

Results: 102 cases were reported from Sept 2017-Oct 18. 65 cases were excluded (duplicate cases, diagnosis date outside study period, case definition not met, clinical data pending) and 37 included.

All patients met SLICC-2012 and 35 patients met ACR-1997. Of the two patients meeting SLICC-2012 but not ACR-1997, one had lupus nephritis on renal biopsy and positive ANA, and the other met the SLICC-2012 hypocomplementaemia criterion.

Of the 35 patients meeting ACR-1997, median age at diagnosis was 12.8 years (interquartile range (IQR) 11.8-14.7 years) with female:male gender of $4.8: 1$ respectively. 24/35 (69\%) were non-Caucasian. Median time from symptom onset to diagnosis was 2 months (IQR 1-6 months). The longest delay was 106 months (patient initially diagnosed with Henoch Schnolein Purpura). 9/35 (26\%) of patients experienced a delay in diagnosis measured by at least one of: established organ damage due to JSLE at diagnosis (5 patients), review by $\geq 1$ paediatric sub-specialist prior JSLE diagnosis (3 patients) or patient not referred despite medical review (6 patients).

The diagnosis was made by or in conjunction with paediatric rheumatology in $21 / 35(60 \%)$ patients. $4 / 35(11 \%)$ patients were diagnosed solely by paediatric nephrology, $2 / 35(6 \%)$ by adult rheumatology, $7 / 35$ (20\%) by general paediatrics and $1 / 35(3 \%)$ by the paediatric infectious diseases team. Of the $12 / 35$ (34\%) patients where diagnosis did not involve a rheumatologist 10 were referred to either adult or paediatric rheumatology and 2 patients were managed solely by paediatric nephrology.

$31 / 35$ (89\%) patients were treated with oral and/or IV steroids and 33/35 (94\%) with hydroxychloroquine. Other treatments used were: mycophenolate mofetil (17/35, 49\%); rituximab (8/35, 23\%); azathioprine (6/35, 17\%); methotrexate $(5 / 35,14 \%)$; cyclophosphamide $(3 / 35,9 \%)$; ofatumumab (1/ $35,3 \%)$; IV immunoglobulin $(1 / 35,3 \%)$; plasmapheresis $(1 / 35,3 \%)$. No patients died within one month of diagnosis.

Conclusion: Diagnosis of JSLE involved paediatric rheumatology in 60\% of cases. Median time to diagnosis from symptom onset is two months but there is significant variation; future work will focus on factors influencing this. Analysis of final data (2 year incidence data) will facilitate estimation of current UK incidence rate and description of factors affecting time to diagnosis.

\section{REFERENCES}

[1] Smith EMD, Foster HE, Gray WK, Taylor-Robinson D and Beresford MW. Predictors of access to care in juvenile systemic lupus erythematosus: evidence from the UK JSLE Cohort Study. Rheumatology 2014;53:557-61

[2] RCPCH. British Paediatric Surveillance Unit. http://www.rcpch.ac.uk/bpsu (accessed 17th January 2019)

Disclosure of Interests: None declared DOI: 10.1136/annrheumdis-2019-eular.1587

\section{SAT0482 TREAT-TO-TARGET STUDY FOR IMPROVED OUTCOME IN POLYARTICULAR JUVENILE IDIOPATHIC ARTHRITIS}

Gerd Horneff, Joachim Peitz, Ivan Foeldvari, Ralf Trauzeddel, Toni Hospach, Tilmann Kallinich, Frank Weller-Heinemann, Ariane Klein, Kirsten Minden. BIKERRegistry, Sankt Augustin, Germany

Background: Evidence suggests that early effective treatment is important to minimize the burden of Juvenile idiopathic arthritis (JIA). We hypothesize that a guided treat to target (T2T) approach as recommended by the German Society for Pediatric Rheumatology (1) is superior to routine care in polyarticular JIA (pJIA) in terms of reaching minimal disease activity and remission after 12 months of treatment.

Objectives: To assess the clinical benefit in subjects with pJIA treated in compliance with national recommendations measured by rates of patients reaching JADAS remission $(\leq 1)$, JADAS minimal disease activity (MDA) $(\leq 3.8)$, JADAS acceptable disease status $(\leq 5.4)$.

Methods: After informed consent, patients with early (disease duration $\leq$ 12 months) and active (JADAS10 > 5.4) pJIA were enrolled. Initially, all patients received methotrexate (MTX). Targets for treatment were defined by the level of improvement and are progressively more rigourous with ongoing treatment. Failure to meet a defined target required treatment modification of specified intervals. The choice of biologic was made by shared decision between the investigator and the patient/parent and not influenced by the protocol. Minimal treatment target defined as recognizable improvement of disease activity (2) was demanded after 3 months, JADAS acceptable disease status at month 6, JADAS MDA at month 9 and JADAS-remission at month 12. T2T Patients were 1:4 matched to a pJIA cohort with unguided therapy documented by the BIKER-registry.

Results: Altogether 62 patients (16 males, 26\%) with non-systemic JIA (48/9 RF negative/positive polyarthritis, 3 extended Oligoarthritis, 1 ERA, 1PsA) were included (mean age $9.4+/-4.8$ years, disease duration $0.5+/-$ 0.6 years). At month 3; 49 (79\%) patients showed JADAS improvement. In $13(21 \%)$ treatment with a biologic was started. At month 6, 45/56 (80\%) reached JADAS acceptable disease. In 6 (11\%) a biologic agent was started. At month 9, 41/48 (85\%) reached JADAS acceptable disease and 38/48 (79\%) reached JADAS-MDA. In $4(10.8 \%)$ a biologic was started and two patient switched biologics.

So far, 52 patients completed 12 months of observation. 4 patients did not start a biologic although mandatory according to protocol and were excluded. JADAS MDA was reached by $39 / 48$ (81\%) and JADAS remis sion was reached by $22 / 48(46 \%)$. Compared to the matched control cohort, upon T2T guidance significant more patients reached JADAS MDA (81\% vs. $60 \%$; odds 2.9[1.3-6.3]; $0=0.006)$ and more patients reached JADAS remission ( $46 \%$ vs. $34 \%$; odds $1.6[0.8-3.0]$; $p=0.15$ ). During the first 12 months of treatment, the T2T approach lead to a significant increase of use of biologics $(46 \%$ vs. $21 \%$; odds $3.2[1.7-6.3]$; $\mathrm{p}=0.0004)$.

\begin{tabular}{|c|c|c|c|c|c|}
\hline & $\begin{array}{c}\text { Baseline } \\
n=62\end{array}$ & $\begin{array}{c}\text { Month } 3 \\
\mathrm{n}=62\end{array}$ & $\begin{array}{c}\text { Month } 6 \\
\mathrm{n}=56\end{array}$ & $\begin{array}{c}\text { Month } 9 \\
\mathrm{n}=52\end{array}$ & $\begin{array}{l}\text { Month } \\
12 n=48\end{array}$ \\
\hline JADAS mean +/-SD & $19.2+/-4.9$ & $7.8+/-5.7$ & $4.3+/-4.8$ & $3.2+/-4.5$ & $2,6+/-2,8$ \\
\hline JADAS- minimal response & n.a. & $79 \%$ & $89 \%$ & $96 \%$ & $96 \%$ \\
\hline $\begin{array}{l}\text { JADAS acceptable } \\
\text { disease }(\leq 5.4)\end{array}$ & $0,0 \%$ & $37 \%$ & $80 \%$ & $85 \%$ & $83 \%$ \\
\hline JADAS MDA $(\leq 3.8)$ & $0,0 \%$ & $34 \%$ & $73 \%$ & $79 \%$ & $82 \%$ \\
\hline JADAS Remission $(\leq 1)$ & $0,0 \%$ & $15 \%$ & $30 \%$ & $44 \%$ & $46 \%$ \\
\hline MTX only & $100 \%$ & $65 \%$ & $62 \%$ & $50 \%$ & $48 \%$ \\
\hline Biologics +MTX & $0 \%$ & $31 \%$ & $33 \%$ & $37 \%$ & $29 \%$ \\
\hline Biologics only & $0 \%$ & $3 \%$ & $5 \%$ & $13 \%$ & $19 \%$ \\
\hline 2nd biologic & $0 \%$ & $0 \%$ & $0,0 \%$ & $4 \%$ & $4 \%$ \\
\hline
\end{tabular}

Conclusion: These data indicate that a T2T concept is feasible and superior. A high rate of patients reached JADAS MDA and JADAS 
remission after 12 months of treatment. Interestingly, about half of patients did not need to be treated with a biologic to reach predefined T2T (2). Thus, the early treatment escalation seems advantageous indicating a window of opportunity to successfully treat polyarticular JIA.

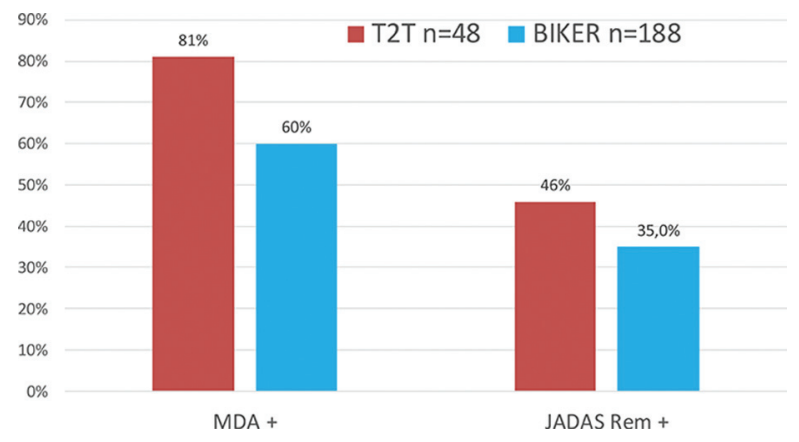

\section{REFERENCES}

[1] Horneff G, et al. Pediatr Rheumatol Online J. 2017; 15:78

[2] Horneff $G$ et Becker I. Rheumatology. 2014;53:1229-34.

Acknowledgement: The authors acknowledge Pfizer Pharma, Germany for an unrestricted grand

Disclosure of Interests: Gerd Horneff: None declared, Joachim Peitz: None declared, Ivan Foeldvari Consultant for: Chugai, Novartis, Ralf Trauzeddel: None declared, Toni Hospach Speakers bureau: Chugai, Roche, Novartis, Tilmann Kallinich Grant/research support from: Novartis, Speakers bureau: Sobi, Roche, Novartis, CLB, Frank Weller-Heinemann: None declared, Ariane Klein: None declared, Kirsten Minden: None declared DOI: 10.1136/annrheumdis-2019-eular.2107

\section{SAT0483 COMPARISON OF THE CLINICAL DIAGNOSTIC CRITERIA AND RESULTS OF THE NEXT GENERATION SEQUENCE GENE PANEL IN PATIENTS WITH PERIODIC FEVER}

Ferhat Demir ${ }^{1}$, Yasemin Kendir Demirkol ${ }^{2}$, Özlem Akgün Doğan², Sezin Canbek ${ }^{3}$, Kübra Ermiş Tekkuş ${ }^{3}$, Nuray Aktay Ayaz ${ }^{4}$, Levent Doğanay ${ }^{5}$, Betül Sözeri' ${ }^{1}$. ${ }^{1}$ University of Health Sciences, Umraniye Training and Research Hospital, Pediatric Rheumatology, Istanbul, Turkey, ${ }^{2}$ University of Health Sciences, Umraniye Training and Research Hospital, Pediatric Genetics, Istanbul, Turkey, ${ }^{3}$ University of Health Sciences, Umraniye Training and Research Hospital, Medical genetics, Istanbul, Turkey, ${ }^{4}$ University of Health Sciences, Kanuni Sultan Suleyman Training and Research Hospital, Pediatric Rheumatology, Istanbul, Turkey; ${ }^{5}$ University of Health Sciences, Umraniye Training and Research Hospital, Genomic Laboratory (GLAB), Health Directorate of Istanbul, Istanbul, Turkey

Background: Autoinflammatory diseases (AID) are a group of hereditary diseases characterised by inflammation periods accompanied with clinical findings such as fever, skin rash, lymphadenopathy, abdominal pain, musculoskeletal symptoms, and with sign of inflammation in the blood. Each disease has own typical clinical findings and they are associated with mutations in specific genes such as in MEFV gene in familial Mediterranean fever (FMF), MVK gene in mevolonate kinase deficiency (MKD), TNFRSF1A gene in TRAPS and NLRP3 gene in cryopyrin associated periodic fever syndrome (CAPS). There are also patients exhibit the incomplete phenotype of a disease or overlap signs of more than one AID. The diagnosis of these patients have difficult and may not be possible by a single target gene analysis. Screening of the periodic fever syndrome (PFS) genetic panel including various AID genes may be beneficial to define the atypical cases.

Objectives: The aim of this study was to compare the phenotypic diagnoses to the genotypic results obtained from the PFS genetic panel, which studied in patients with phenotypic findings of AIDs except the FMF such as MKD, TRAPS and CAPS.

Methods: This is a prospective study and conducted between June 2016 and December 2018. Patients who met "The Eurofever clinical diagnostic/ classification criteria"* for MKD, TRAPS and CAPS were included in the study. Next-generation sequencing (NGS) analysis was performed including 8 genes (MEFV, MVK, NLRP3, NLRP12, TNFRSF1A, TNFRSF11A, LPIN2 and PSTPIP1) in 37 patients with phenotypic findings of MKD, TRAPS or CAPS. The patients phenotypic preliminary diagnoses and genotypic results were compared.
Results: Thirty seven patient included in the study who met the clinical diagnostic criteria for MKD, TRAPS or CAPS. As a result of clinical signs; 19 patients were diagnosed with MKD, 10 were TRAPS and 8 were CAPS

In the PFS genetic screening panel of 19 patients with phenotypic diagnosis of MKD; disease causing mutation was found in 8 patients in MVK gene, in 3 patients in MEFV gene, in 2 patients in NLRP12 gene and in 1 patient in TNFRSF1A gene. No pathogenic mutation was shown in 3 patients and genetic variants of uncertain significance (VUS) in different genes were shown in two patient.

In the result of PFS panel of 10 patients with phenotypic diagnosis of TRAPS; disease causing mutation was found in 5 patients in MEFV gene and in 2 patients in TRAPS gene. No pathogenic mutation was identified in 3 patients.

In 8 patients who were diagnosed phenotypically as CAPS; disease causing mutation was found in 3 patients in NLRP3 gene, in 2 patients in NLRP12 and in one patient in MEFV. No mutation was detected in remaining two patient.

The final diagnosis was made by both phenotypic and genotypic data. In 37 patients (phenotypically 19 MKD, 10 TRAPS, 8 CAPS), respectively; 8 had MKD, 9 had FMF, 3 had CAPS, 3 had TRAPS, 4 had FCAS2 and 10 had undifferentiated PFS.

Conclusion: In our study, we have shown that clinical diagnostic criteria may not always be sufficient to establish the correct diagnosis. In case of clinical suspicion or in the presence of more than one autoinflammatory disease findings, NGS analysis may help to determined the diagnosis.

Acknowledgement: We are grateful to all participating children and their families. Ethics committee approval was received from the Institutional Review Board of Umraniye Training and Research Hospital. All the participants and their legal guardians were informed, and their written consent was obtained. Disclosure of Interest: None Declared

Disclosure of Interests: None declared

DOI: 10.1136/annrheumdis-2019-eular.7378

\section{SAT0484 \\ QUALITY OF LIFE IN JUVENILE IDIOPATHIC ARTHRITIS- ASSOCIATED UVEITIS: DO WE NEED A NEW ASSESSMENT QUESTIONNAIRE?}

Mario Sestan ${ }^{1}$, Danica Grguric ${ }^{1}$, Maja Sedmak ${ }^{1}$, Nastasia Kifer ${ }^{1}$, Marijan Frkovic ${ }^{1}$, Sanja Peric ${ }^{2}$, Kristina Potocki ${ }^{3}$, Nenad Vukojevic ${ }^{2}$, Marija Jelusic ${ }^{1} .{ }^{1}$ University Hospital Centre Zagreb, University of Zagreb School of Medicine, Department of Paediatrics, Zagreb, Croatia; ${ }^{2}$ University Hospital Centre Zagreb, University of Zagreb School of Medicine, Department of Ophthalmology, Zagreb, Croatia; ${ }^{3}$ University Hospital Centre Zagreb, University of Zagreb School of Medicine, Department of Radiology, Zagreb, Croatia

Background: Uveitis, the most common extra-articular manifestation in juvenile idiopathic arthritis (JIA), occurs in $10-20 \%$ of patients. Although prognosis of uveitis within JIA (JIA-U) improved, complications still cause severe impairment of visual function in $25-33 \%$ of children that affects their psychophysical and psychosocial development and quality of life (QoL).

Objectives: To study the QoL and it's dimensions in children suffering from JIA-U as well as to investigate is there any difference in childhood's and parent's perception of disease between the group of children with JIA-U and children with JIA without uveitis.

Methods: The study included 42 children with JIA and their parents Patients were divided into two groups. The first consisted of 21 children with JIA-U and the second of 21 children with JIA and no uveitis. Both groups of patients and their parents filled the Juvenile Arthritis Multidimensional Assessment Report questionnaire (JAMAR) for monitoring and assessing the health status of children with JIA. The variables used to test differences were: QoL, functional ability, pain level, disease activity estimation, and current emotional state of the child. The significance of differences between groups of children and parents was verified by the independent-samples t-test. The Pearson correlation coefficient was used for measurement of the strength of the linear relationship between variables.

Results: There were no statistically significant differences in the JIA-U group and the control group in either of the examined variables. Although there is a tendency of higher scores in children with JIA-U, which indicates their worse functioning, higher pain intensity and worse current emotional state, these differences were not statistically significant. Two groups did not differ significantly in the assessment of their own overall functional ability, which was associated with experienced pain intensity. Stronger pain intensity was associated with dysfunction $(r=0.642$, $p$ 